\title{
A Hybrid Optical-Radio Wireless Network Concept for the Hospital of the Future
}

\author{
Iqrar Ahmed, Timo Kumpuniemi and Marcos Katz \\ Centre for Wireless Communications, \\ University of Oulu, Finland \\ firstname. lastname@oulu.fi
}

\begin{abstract}
In this paper we consider a hybrid optical-radio wireless network as a flexible, secure and high-performance communication network for the hospital of the future. The network consists of access points as well as devices capable to communicate using optical and radio technologies. Wireless body area networks (WBAN) are one of the key elements needed to achieve the desired goals. Within the proposed hybrid network, we extend the conventional WBAN concept to a more generic and highly flexible, reconfigurable optical-radio wireless body area network (RORWBAN). Ultimately, the concept of hospital of the future aims at enhancing healthcare while using thoughtfully and efficiently hospital resources. Wireless networks are one of the key enables of tomorrow's hospitals. However, spectral usage, security, safety and privacy issues of current radio-based wireless networks could undermine the attractiveness of these networks for future healthcare scenarios. The proposed solution, combining optical and radio transmission results in a network that can adapt itself in short- and long-terms to the changing communications requirements of hospitals. In principle, a hybrid network can tackle interference, exposure, privacy and other key problems of conventional networks. The proposed system could intelligently reduce the exposure to radiation and generation of radio interference, while parallel opticalradio transmission could ensure secure, reliable and high capacity communications, using efficiently the available spectrum. In this paper, the overall concept will be presented, and the possible operation scenarios and applications will be introduced too. Finally, we discuss the challenges of hybrid optical-radio WBAN for future hospitals.
\end{abstract}

Keywords: Reconfigurable networks, Visible light communications, Hybrid wireless networks, Hospital technology, Bio signals, Optical wireless communications, Wireless body area networks.

\section{Introduction}

There is currently a growing interest in developing concepts for the hospital of the future $[1,5,6]$. This is a multi-disciplinary development, including not only technology aspects but also medical and nursing sciences, architecture, management, etc. The role of technology in this concept is essential, as it makes possible and actively supports advanced healthcare methods, efficient use of hospital resources, exploitation of data, 
efficient management, automation, etc. In turn, most of these desired characteristics for tomorrow's hospital rely on the concept of connected hospital, a notion allowing connectivity between all key stakeholders of the hospital, e.g., patients, health-care personnel, equipment, data centers, other hospitals, IoT nodes, etc. In most of the cases, wireless connections are preferred, for the several well-known advantages of wireless networks. The key to the future hospital is to provide quality healthcare to all, regardless of the limited resources and increasing healthcare requirements and costs. The future hospital paradigms may include self and remote monitoring, mobility, hazard-free monitoring, data protection and anonymity. In the future, different types of wireless networks will be deeply integrated in hospitals and health-care centers.

Radio-technology has been the dominant approach to provide wireless connectivity in virtually any possible scenario. However, spectrum congestion, interference, security, privacy and safety issues, energy efficiency and others, are still challenges of radio-based systems. In recent years, optical wireless communications technology has emerged as a viable way to transmit information wirelessly. Visible light communications exploit the lighting infrastructure of solid-state light sources (e.g., white light-emitting diodes) to provide light-based wireless connectivity. Light-based wireless systems complement effectively radio technologies, as the key advantages of the former can counteract the most important drawbacks of the latter, particularly the aforementioned spectrum shortage, security, safety, security and interference. Recently, hybrid optical-radio networks have been proposed aiming at exploiting the flexibility and performance of both network approaches [2-3]. This hybrid network is particularly attractive for sensitive environments such as hospitals, as the combined radio-optical network can be flexibly configured to adapt to particular scenarios and fulfill their associated requirements. A hospital equipped with hybrid optical-radio wireless networks will fulfill the most demanding requirements for performance, security and safety. In this paper we discuss this concept in more detail.

This paper is organized as follows. In Chapter 2 we discuss the possible wireless communication networks for future hospital. Chapter 3 introduces the proposed hybrid optical-radio network, operating scenarios and applications within the hospital. Finally, in chapter 4 we discussed the challenges associated with the proposed system and in chapter 5 we concluded our study.

\section{Wireless Networks for Tomorrow's Hospitals}

In this section, we briefly describe the most important types of wireless networks exploited by hospitals and health-centers, namely Wireless Body Area Network (WBAN), Wireless Local Area Networks (WLAN) and Wireless Wide Area Networks (WWAN). Figure 1 illustrates these networks from the hospital standpoint. In this paper, particular attention will be paid to the short-range networks (i.e., local coverage) and their relationship to hospitals. 


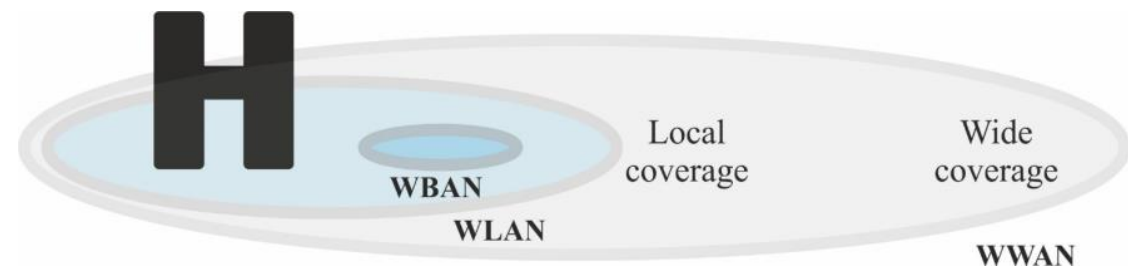

Fig. 1. Key wireless networks supporting the operation of a modern hospital: Wireless body area networks (WBAN), wireless local area networks (WLAN) and wireless wide area network (WWAN).

\subsection{Wireless Wide Area Networks (WWAN)}

Wireless wide area networks refer to wide coverage cellular networks. From a hospital perspective, large cells, e.g., macro- and micro-cells, cover large areas (well outside the hospital itself), supporting also mobility. This is needed to support remote healthcare and healthcare on the move. Pico- and femto-cells can be also deployed inside hospitals to increase capacity and enhance service.

\subsection{Wireless Local Area Networks (WLAN)}

Wireless local area networks can provide cost-efficient high performance local access to most of the typical hospital scenarios. These standardized general-purpose wireless networks have and will have an important role in hospitals, as they provide reliable, low-cost and locally-controllable access to a variety of hospital scenarios, bridging healthcare equipment, people, computers, mobile devices, IoT nodes and others.

\subsection{Wireless Body Area Networks (WBAN)}

During the past years, we have witnessed a rapid increase in the adoption of internet of things (IoT) technology. IoT sensors can be installed on a variety of objects and devices, such as body-worn devices, home appliances, environmental stations, buildings, vehicles, to wirelessly monitor and control these elements through the internet infrastructure. Currently, huge global efforts are being made in the development and implementation of the fifth generation $(5 \mathrm{G})$ technologies, which will expand the number of IoT sensors into a totally new level, also allowing interoperation across different wireless networks. A WBAN is a close-range wireless network of nodes deployed on and in the human body. Nodes can include sensing and processing units, transceivers, and other functionalities. In a WBAN, one or more nodes can serve as access points or gateways, allowing exchange of data between the WBAN nodes and external devices. WBANs can be classified in on-body networks where the nodes are installed on the surface of the human bodies. In off-body cases, nodes are located in close vicinity of the humans (e.g., pockets, bags, etc.) while in an in-body cases nodes are placed inside the human bodies (e.g., implants). In body-to-body WBANs, nodes can exchange data between two or several individuals [11]. As population is ageing, the adoption of WBANs can provide a cost-effective solution to ease up the problem of monitoring health conditions, reducing costs and increasing the work efficiency of medical staff. 
In fact, local networks enable to monitor the health of the patients in hospital wards, waiting rooms and other premises of the hospitals and health centers without the constant presence of the medical staff. Cellular networks extend these capabilities when patients are outside the hospital, so remote monitoring can take place on the move, at home, etc. WBANs can also find applications in sports and training field, providing readily information on the effectiveness of training, for example. Moreover, WBANs can be used to monitor wellbeing, for instance gathering information on sleep and day activity. Today, a number of different wireless technologies exist to be applied in medical scenarios, as discussed in [12]. They can be used to transmit body functions and parameters constantly through numerous sensors already commercially available [12]. However, these systems are based on radio frequency (RF) communications. Several systems operating at the same location might create interference that can eventually disrupt the data transmission [12].

\subsection{Visible Light Communications (VLC)}

Visible light communications is a relatively new wireless communication technology that exploits solid-state lighting infrastructure to create light-based wireless links. In recent years, a great deal of research and development on VLC was carried out, and VLC was shown to be an attractive wireless communication approach for indoor, outdoor, vehicular and underwater scenarios. The principal advantages of VLC are the large and unregulated available bandwidth, the inherent security and privacy of optical systems, and due to the zero-radio emission, the suitability of VLC to both interferencesensitive environments and no radio exposure use cases. Fig. 2 illustrates the basic structure of VLC transceivers using visible light for downlink and infrared light for uplink.

The use of VLC systems in medical environments has been proposed by several authors. Cahyadi et al [7] demonstrated a system where the clinical data from three bio sensors can be transmitted via VLC uplink. Predefined headers were added to each sensor to differentiate their signals at the receiver end. Authors reported minor interference in received signal caused by the flux of ceiling. The clinically interesting frequency range of mostly bio signals starts from DC and can be up to tens of Hertz, the bio signals might see several artefacts such as interference due to multiple sensors working at the same time when transmitted on RF. VLC presents an alternative solution for transmitting bio signals where these are less susceptible to artifacts. Dhatchayeny et al. [8] presented a study in which an EEG signal can be transmitted efficiently on VLC without any loss, as might be the case in radio transmissions. EEG signal comprises of multiple frequencies describing the ongoing activities in different parts of brain, thus transmitting each frequency separately is critical for proper diagnose. Authors proposed a solution of duplicate parallel optical stream of EEG signal on RGB spectrum, the received bio signals are identical when compared. Using three separate light wavelengths ensures zero interference in transmission. In [9], a concept of combining VLC and power line communications (PLC) in hospital for numerous purposes have been presented. PLC exploits the power lines to modulate the existing light resources for communications. Authors addressed the benefits VLC and PLC as a 
backbone of hospitals for transmitting the medical data. Following the evolution of ehealth, the use of electronic patient records has been in common practice around the globe, where patients' clinical data is stored in a cloud. This is hugely advantageous to patients and hospitals, as patient data can be accessed from anywhere. However, due to the huge amount of data, high link capacity is crucial for efficient operation. Moreover, privacy and security are required for this type of communications. VLC can be used as an optimal solution in these applications. Authors demonstrated a $48 \mathrm{Mbit} / \mathrm{s}$ data link supporting data transmission, and connecting both patients and clinicians, as in the connected healthcare paradigm. Other proposed application scenarios could be operating smart devices, e-conferencing and it could also help clinicians to give light therapy to patients suffering depression disorders. Similar studies i.e., on VLC for transmitting bio signals can also be found in [10].

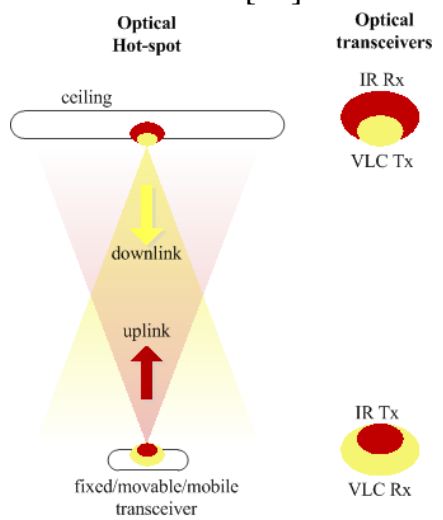

Fig. 2. Basic realization of uplink and downlink VLC transceivers.

\subsection{Hybrid Networks}

For more than a century, radio-technology has been the dominant approach to provide wireless connectivity in virtually any possible scenario. However, spectrum congestion, interference, security, privacy and safety issues, energy efficiency and others, are still challenges of radio-based systems. In recent years, optical wireless communications technology has emerged as a viable way to transmit information wirelessly. Light-based wireless systems, such as VLC, complement effectively radio technologies, as the key advantages of the former can counteract the most important drawbacks of the latter, particularly the aforementioned spectrum shortage, security, safety, security and interference. In recent years, hybrid optical-radio networks have been proposed aiming at exploiting the flexibility and performance of both network approaches [2-3]. Hybrid networks are particularly attractive for sensitive environments such as hospitals, as the combined radio-optical network can be flexibly configured to adapt to particular operation scenarios and their associated requirements. Figure 3 illustrates the key stakeholders of tomorrow's hospital, connected by both local radio and optical wireless networks. Patients, medical staff, equipment, computers, mobile devices, distributed sensors and actuators will all be wirelessly networked in the hospital of the future. The highly complementary component networks bring redundancy to the system, which can 
be exploited in different manners. Indeed, an optical-radio network will guarantee flexibility, reliability, high performance and efficient utilization of resources.

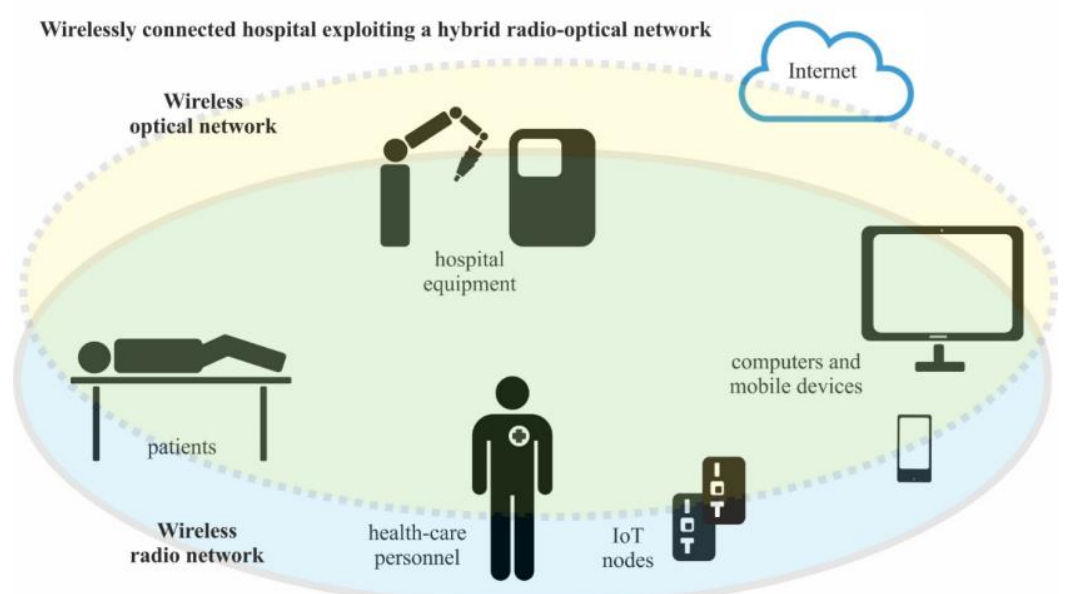

Fig. 3. Connected hospital of the future: Exploiting radio and optical wireless networks.

\section{A Reconfigurable Optical-Radio Wireless Network for the Hospital of the Future}

In this section, we present an optical-radio wireless network for future hospitals. Although the concept of the future hospitals has been discussed in many studies, the use of optical and radio links for medical data transmission has not be considered from this forward-looking perspective. Based on the hybrid optical-radio wireless network principle discussed in the previous section, we define a reconfigurable hybrid wireless network aimed for the hospital of the future. The network consists of a) one or more reconfigurable hybrid access points and $\mathrm{b}$ ) one or more reconfigurable wireless body area networks. The term reconfigurable refers here to the capability of the network to dynamically change the transmit and receive modes. More particularly, reconfiguration allows to select optical and radio modes for sending information between transmitting and receiving ends. Different mode selections will be defined later in the paper.

\subsection{Reconfigurable Optical-Radio Access Points}

A reconfigurable optical-radio access point consists of hybrid transceiver with different operating modes. Downlink (DL) and uplink (UL) can be independently selected to transmit and receive using in each case either optical or radio links. This gives rise to four operating modes, namely a) DL optical, UL optical; b) DL optical, UL radio; c) DL radio, UL optical and d) DL radio, UL radio. In addition, the system can be configured to exploit diversity, that is both transceivers are simultaneously used. A 
reconfiguration algorithm selects the most appropriate operating mode based on a number of inputs, such as optical/radio channel conditions, type of scenarios, service requirements, service provider/user decisions, local policies, etc. Note that reconfiguration is dynamic, and changes could occur over short-term periods (e.g., in response to sudden channel blockage) and also controlled over long-term periods, as such dictated by the operating scenarios and local policies, for instance. Figure 4 illustrates the concept of reconfigurable optical-radio wireless networks, particularly devised for tomorrow's hospitals.

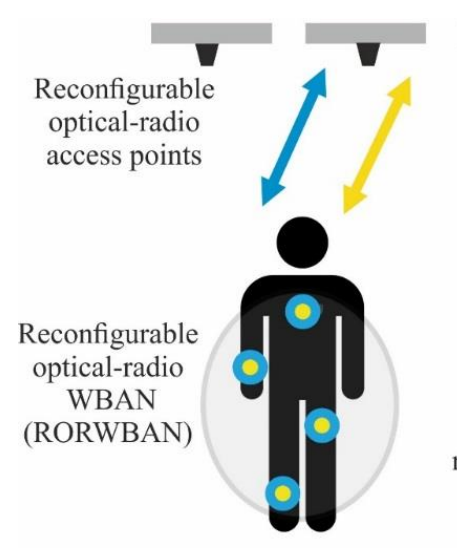

Reconfigurable optical-radio wireless networks for the hospital of the future
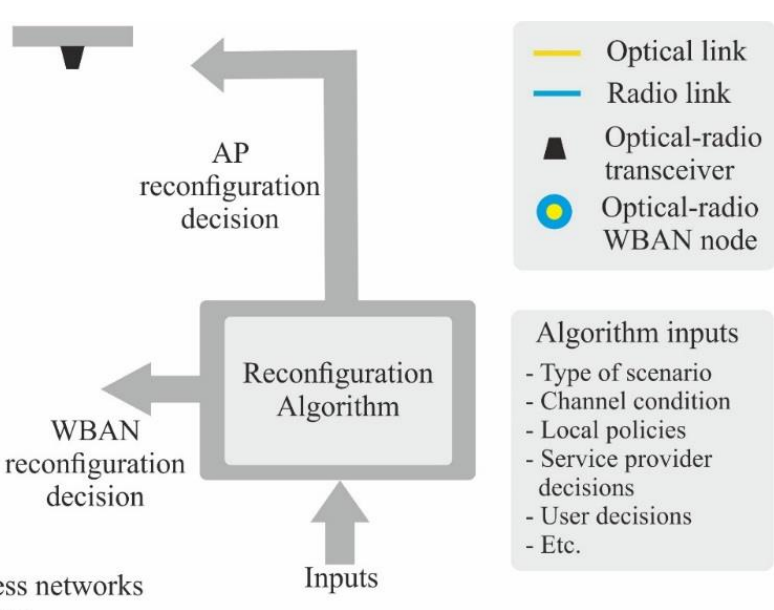

Fig. 4. Wireless communications networks for the hospital of the future:

Reconfigurable Optical-Radio Access Points and Reconfigurable Optical Radio WBANs.

\subsection{Reconfigurable Optical-Radio Wireless Body Area Network (RORWBAN)}

We extend the conventional WBAN concept to consider a more generic network where some or all network nodes can be independently reconfigured in the same fashion as discussed for access points. That is, a generic node of the WBAN can be controlled to operate in different possible modes, exploiting optical and radio links. This defines a reconfigirable optical-radio wireless body area network, RORWBAN. In general, a RORWBAN has $N$ nodes, out of which $M$ are hybrid (optical/radio) and the remaining $(N-M)$ nodes are single-technology (either radio or optical). Eventually, all the nodes can be hybrid $N=M$ (fully hybrid WBAN). Figure 4 depics a RORWBAN where $N=$ $M=4$. Note that if $N=1$, the RORWBAN is reduced to a simple single-node case, as analyzed in our previous work $[2,3]$.

The RORWBAN can be configured dynamically (on the fly) according to the prevailing instantaneous conditions as well as some general environmental/context/policy situation (typically but not necessarily fixed), in the same way as discussed for the hybrid accerss points. Note that power consumption could also be used as a mode selection criterion. The reconfiguration algorithm selects the appropriate operating mode for the WBAN, action done jointly with the access point mode selection. Implementation of a hybrid node could be optimized to support 
simplicity and cost reduction. In such a case the node should use as much as possible common communicationsl blocks, only transmitting/receiving ends could be changed according to the selected optical/radio mode. Nodes could also be implemented as different paralel optical and radio branches, and the selection will then focus on the complete branch.

\subsection{Operating Scenarios/Applications}

Unique VLC features, such as high data rate support, inherent security, less interference to bio signals and to sensitive equipment, hazard-free transmission, cost-effective implementation (existing PLC and light resources infrastructure can be reused), make VLC an attractive solution for hospital wireless connectivity. Added to this, is the flexibility, simplicity and performance of radio networks. Combining both technologies as suggested here will help to fulfill the communications requirements of the hospital of the future. Key objectives of future hospitals include enhancing the healthcare quality, management i.e., self and remote, data security and anonymity. We consider next two attractive operating scenarios for future hospitals.

Reconfigurable Optical-Radio Wireless Networks within the Hospital: We consider first the use of the hybrid wireless networks in different hospital scenarios, and for a variety of requirements. By maximizing the use of optical links, the proposed architecture provides an efficient mean of reducing a) radio exposure and b) generated interference. In other words, whenever possible the traffic is sent through optical wireless communications. Some potential applications of proposed RORWBAN within a future hospital are discussed.

$R F$-restricted areas/coexistence. The use of RF devices is prohibited in specific hospital areas e.g., radiology and CT labs since radio can interfere with other devices. This is also the case in heavily guarded facilities such as in oncology and catheterization labs, where radio interference is also an issue. In such situations, the wireless network can be configured a fully optical, so sensors data can be safely transmitted despite radio restrictions. In highly sensitive environments, as, e.g., operation rooms or premises with high fidelity medical measuring and treatment equipment, radio communications may be an unwanted technology as such. Optical links offer a simple solution, as well as protection against data leakage through eavesdropping. At homes, this is a convenient way to create a controllable, data transmission safe environment.

Off-loading. While performing tests in the aforementioned labs, a large number of sensors is used to monitor the physiological state of the subject which is essential for proper diagnosis. Transferring this information using conventional radio could easily consume prohibitive amounts of bandwidth. Data could be here partitioned and transmitted in optical and radio links, and eventually only optical links could be utilized.

Support of mobility and localization. Another potential advantage is indoor positioning, exploiting both optical and radio infrastructure for more accurate results. The ubiquity of optical and radio networks allows the data transmission while the subject is displaced from ward or moving. This could also help clinical robots to be 
remotely positioned and controlled when performing different tasks in highly hazardous areas. In other words, a hazard-free environment can be possible in future hospitals.

Data security. Optical communications provide inherently secure communications as the signal is spatially confined in the area of operation. This feature could help to secure the data since the light waves cannot breach the walls or windows, so it secures the data to be hacked from outside. However, a hybrid network allow protection to a higher degree. We proposed a novel way of data encryption. Using the proposed hybrid network, we can divide and transmit the data in two domains, radio and optical. In order to decode the received data both parts need to be present. Clearly the optical component is very local, whereas the radio part could be transmitted either by local or wire area (cellular) networks.

Energy efficiency/saving. The hybrid network can be configured in a low-power mode, where the selection will depend on the power consumption profile of the optical and radio implementations, among others.

High reliability, high data throughput. The multiplicity of nodes (RORWBAN) and possible links (optical/radio) creates a number of diversity branches that can be readily exploited to enhance reliability of the communications. The same principle can be applied to achieve high data rates, by using a number of parallel data pipes to transfer information.

Reconfigurable Optical-Radio Wireless Networks outside Hospital (Remote Hospital): An important scenario of the future hospital is that healthcare services are not confined exclusively to hospital territory. Remote and self-care in healthcare sector have strengthen this scenario, our proposed system allows homes to be turned into hospitals. This could help to reduce pressure on healthcare personnel, reduce costs and improving wellbeing of patients, without compromising the healthcare quality. The considered hybrid network can be used for monitoring the subjects from home. WBAN sensors using both optical and radio links can accommodate the data loss in case either link fails, as a vertical handover mechanism (VHO) switches the links whenever a fail occurs. Since a remote location working as a hospital extension is not regulated (e.g., a hospital is a controlled environment), a conventional radio-based WBAN is more likely to be interfered (or create interference to near-by equipment). Again, optical systems or a combination of radio and optical approaches can eliminate or drastically reduce possible interference problems with surrounding equipment.

\subsection{Operating Modes}

In past recent years, hybrid optical-radio networks have been demonstrated aiming at exploiting the flexibility and performance of both network approaches [2,3]. These hybrid networks utilize potential vertical handover (VHO) techniques for allocating network at predefined rules. Based on those studies our proposed hybrid network can also switch between five different operating modes. These modes are: 
- Mode 1, fully optical. Both uplink and downlink are visible light channels in this case, the data is transmitted through LED driven by modulated signals.

- Mode 2, fully radio. In this case RF uplink and downlink are utilized to transmit the data between the source and the sink.

- Mode 3, hybrid 1. In Hybrid 1 mode the uplink is established using optical channel whereas all the downlink data can be transmitted through RF link.

- Mode 4, hybrid 2. Hybrid 2 mode is the opposite of Hybrid 1 where the uplink is established using RF channel and all the downlink data can be transmitted through optical link.

- Mode 5, co-operative. In co-operative mode both RF and optical channels can be used as complementary to each other, and also simultaneously. VHO techniques allows networks to switch between both modes in case of failure of either link.

Hybrid modes 1 or 2 can be used when there is a large traffic imbalance between uplink and downlink, in off-loading situations, and due to uneven resources availability.

The considered system can select any mode based on the local policies defined by operator or user according to the ground situations as well as some predefined rules such as VHO strategies could also make selections among different operating modes discussed above.

\section{Challenges}

Since VLC is relatively new compared to well-established radio communications, numerous challenges need to be addressed, and current research in WBAN using radio could help to design and enhance the capabilities of RORWBAN.

Sensor: The sensor package should match or fit the human body and each sensor should be kept small. Since our proposed system uses both radio and optical transmitter and the overall size also determines the weight carried, novel IC packaging is crucial for RORWBAN.

Throughput: Though very high data rate of up to $1 \mathrm{Gbit} / \mathrm{s}$ has been achieved with OWC [13], the SNR could deteriorate progressively in non-LOS communications or when obstructed partially.

Reconfiguring mechanism/Complexity: Devising a fast and flexible, yet robust control algorithm to reconfigure both access points and RORWBAN could be challenging task, as the number of input parameters could be significant large, and their interrelationship complex. The use of machine learning and AI could help to design an effective decision-making mechanism.

Energy harvesting: To be practically attractive, the expected power consumption of nodes is expected to be less than $1 \mathrm{~mW}$ [14]. In practice, however, the battery-powered WBAN sensor consumption should be as minimum as possible, the overall size of sensor and circuitry play an important role in battery life. For our proposed system, energy harvesting could be a solution to enable sensor to work for longer durations. 
Note that light could not only power up the sensor but also convey data information as in VLC.

Security: Data security is another key challenge, we proposed partial parallel radiooptical transmission of data. In that case, the data from either links is not meaningful unless the receiver obtains the other part of data. Coding for parallel radio-optical transmission is a challenging task.

Integrity and Intelligence: The integrity of received signal is of utmost importance, any missed or falsely transmitted signal could risk a human life. Thus, the read out and sensing errors need to be addressed briefly. The intelligence for a complex system as RORWBAN is critical not only to handle network links and to pre-process the bio signals before transmission. The proposed system could work in different operating modes as mentioned above, it requires an embedded intelligence to switch the links in case of failure. Also, the pre-processing intelligence of bio signal before transmitting is to remove artefacts embedded into them which could result in background noise or low SNR.

\section{Conclusions}

In this paper, we proposed a novel architecture for wireless communication networks particularly focused for operating scenarios of future hospitals. Our proposed system exploits the relatively new VLC and conventional radio communicational techniques to build a flexible, secure and radiation/interference safe network which can satisfy the communication requirement of the hospital of the future. The reconfigurable nature of the system could provide a platform to implement and test novel resource allocation strategies for further enhancement of system intelligence. Operating scenarios and modes are discussed in detail and could help in self and remote healthcare diagnostics. The proposed system utilizes the existing solid-state-lighting-based structure for VLC thus can be adopted and could act as a backbone for data traffic connecting the hospital on a single highway. Several challenges are to be overcome to build and embed the proposed system into the hospital environment, but the flexibility, security and user safety features make the proposed system an integral part of the future hospitals.

\section{References}

1. Future Hospital OYS 2030, http://www.oys2030.fi/english.php

2. Saud, M. S., Chowdhury, H., \& Katz, M. (2017, March). Heterogeneous Software-Defined Networks: Implementation of a Hybrid Radio-Optical Wireless Network. In Wireless Communications and Networking Conference (WCNC), 2017 IEEE (pp. 1-6). IEEE.

3. Saud, M. S., \& Katz, M. (2017, May). Implementation of a Hybrid Optical-RF Wireless Network with Fast Network Handover. In European Wireless 2017; 23th European Wireless Conference; Proceedings of (pp. 1-6). VDE.

4. Hall, P.S., Hao, Y.: Antennas and propagation for body-centric wireless communications. 2nd edn. Artech House, Norwood, MA, USA (2012). 
5. Jakob E. Bardram, Hospitals of the Future - Ubiquitous Computing support for Medical Work in Hospitals, UbiHealth 2003: The 2nd International Workshop on Ubiquitous Computing for Pervasive Healthcare Applications

6. White Paper: The Hospitals of the Future, Advanced Wireless Technology Group, UK, Available online at http://awtg.co.uk

7. Cahyadi, W.A., Jeong, T.I., Kim, Y.H., Chung, Y.H. and Adiono, T., 2015, November. Patient monitoring using visible light uplink data transmission. In Intelligent Signal Processing and Communication Systems (ISPACS), 2015 International Symposium on (pp. 431-434). IEEE.

8. Dhatchayeny, D.R., Sewaiwar, A., Tiwari, S.V. and Chung, Y.H., 2015, May. EEG biomedical signal transmission using visible light communication. In Industrial Instrumentation and Control (ICIC), 2015 International Conference on (pp. 243-246). IEEE.

9. Ding, W., Yang, F., Yang, H., Wang, J., Wang, X., Zhang, X. and Song, J., 2015. A hybrid power line and visible light communication system for indoor hospital applications. Computers in Industry, 68, pp.170-178.

10. Tan, Y.Y., Jung, S.J. and Chung, W.Y., 2013, July. Real time biomedical signal transmission of mixed ECG signal and patient information using visible light communication. In Engineering in Medicine and Biology Society (EMBC), 2013 35th Annual International Conference of the IEEE (pp. 4791-4794). IEEE.

11. Karvonen, H., Hämäläinen, M., Iinatti, J., Pomalaza-Ráez, C.: Coexistence of wireless technologies in medical scenarios. In: Proceedings of the 2017 European Conference on Networks and Communications (EuCNC), 1-5, IEEE, Oulu, Finland (2017).

12. Karvonen, H., Mikhaylov, K., Hämäläinen, M., Iinatti, J., Pomalaza-Ráez, C.: Interference of wireless technologies on BLE based WBANs in hospital scenarios. In: Proceedings of the 2017 IEEE 28th Annual International Symposium on Personal, Indoor, and Mobile Radio Communications (PIMRC), 1-6, IEEE, Montreal, Canada (2017).

13. G. Cossu, W. Ali, R. Corsini, and E. Ciaramella, "Gigabit-class Optical Wireless Communication System at Indoor Distances (1.5-4 m), Optics Express, vol. 23, no. 12, pp. $15700-15705$, Jun 2015.

14. P. D. Mitcheson, E. M. Yeatman, G. K. Rao, A. S. Holmes, T. C. Green, "Energy Harvesting From Human and Machine Motion for Wireless Electronic Devices," Proceedings of the IEEE, vol. 96, pp. 1457-1486, 2008. 